\title{
Palliative Care in Emergency Medicine. What Are We Missing?
}

\author{
Lauren Nigolian'1, Raymund Gantioque ${ }^{1}$, Joshua Dexheimer ${ }^{2}$ \\ ${ }^{1}$ Department of Nursing, California State University of Los Angeles, Los Angeles, CA, USA \\ ${ }^{2}$ Department of Kinesiology, Azusa Pacific University, Azusa, CA, USA \\ Email:1lacues@calstatela.edu, jdexheimer@apu.edu,rgantio@calstatela.edu
}

How to cite this paper: Nigolian, L., Gantioque, R. and Dexheimer, J. (2019) Palliative Care in Emergency Medicine. What Are We Missing? Open Journal of Emergency Medicine, 7, 8-15.

https://doi.org/10.4236/ojem.2019.71002

Received: November 30, 2018

Accepted: March 26, 2019

Published: March 29, 2019

Copyright () 2019 by author(s) and Scientific Research Publishing Inc. This work is licensed under the Creative Commons Attribution International License (CC BY 4.0).

http://creativecommons.org/licenses/by/4.0/

\section{cc) (i) Open Access}

\begin{abstract}
Patients present to the emergency department with critical and complex medical conditions that require a broad scope of medicine to achieve patient outcomes. Emergency medicine physicians are recognizing the importance and positive outcomes that arise when palliative care teams are consulted in the emergency room. Today, medical schools, residency programs, and emergency departments are requiring palliative care curriculum apart of their education. However, there continues to be a gap in early initiation of palliative care in emergency medicine. Nurse practitioners are becoming pivotal in the emergency department, and patients are considering them key providers in their medical journey. The role of an advanced practice nurse in an emergency room may be optimal for the early onset of palliative care consultation. This manuscript examines current knowledge that explores the background of palliative care, the current practice in the emergency department, the positive outcomes and gaps that still exist today, and the increasing role of an advanced practice nurse in the emergency room and their impact on palliative care initiation.
\end{abstract}

\section{Keywords}

Emergency Department (ED), Emergency Room (ER), Palliative Care

Consultation (PCC), Palliative Care (PC), Nurse Practitioners (NPs)

\section{Case Study}

Mr. Smith, a 75-year-old man, has been admitted multiple times to the critical care unit because of his recurring COPD and heart failure exacerbations. Mr. Smith suffers from respiratory failure secondary to heart failure and has been in and out of the hospital for the past 6 months, requiring aggressive management 
and treatments during each admission. He remains a full code, and his wife continues to tell the physicians he "wants everything done". With life threatening diseases such as progressive COPD and heart failure, at some point, these diseases will lead to life support or death. If Mr. Smith does recover, what will that look like? What will his quality of life be? As Mr. Smith continued aggressive care in the ICU, one of the critical care nurses caring for him suggested a palliative care consultation. The palliative care team was finally consulted and was able to meet with the patient and his wife to learn about the couple's values, beliefs, and goals of care. The palliative care team also assessed their perceptions and ability to handle sensitive information. For example, Mr. Smith expressed his tiredness of fighting his diseases and wanting to go home and rest. On the other hand, Mrs. Smith expressed her concern: if her husband died, she wouldn't know how to live her life alone without her husband. Although the end of this case study is unknown, palliative care teams serve as another multidisciplinary aspect of care for patients. Patients' decisions after palliative care consultations are not predictable, but there is evidence that indicates palliative care teams help patients maximize their quality of life, improve their pain, and in some instances help patients live longer [1].

\section{Introduction}

As the population ages, many patients will present to the ED with serious and complex medical conditions that require a broad scope of medicine to achieve patient outcomes [2]. The ED is where most patients seek urgent care for acute, chronic, and malignant diseases. ED providers are trained to provide acute management and treatment plans aimed at preserving patients' lives. Until recently, there has been a shift in education for acute aggressive interventions to patient-goal assessment among emergency medicine providers [2]. This large movement has focused on educating emergency providers on end of life care and consulting palliative care (PC) teams in the emergency room (ER). Although there have been benefits for consulting PC teams in the ED, there still remains a lack of consensus among emergency staff in regard to this topic. The ED is a fast-paced environment where providers are trained to implement aggressive and acute treatments to patients that enter the ER. Emergency physicians may be focused on lifesaving treatments for the patients and disposition to appropriate units, not considering the need for a palliative care consultation (PCC). With the appropriate timing, education, palliative care models, and the uprising role of a Nurse Practitioner (NP), recent studies demonstrated the positive outcomes of PC in the ED, such as improved patient outcomes, family and patient communication, and reduction in hospital costs and patient's length of stay [2].

\section{Definition of Palliative Care}

PC was once defined as "the relief of symptoms including pain that interferes with the quality of life" [2]. Now according to World Health Organization, PC is 
defined as "an approach that improves the quality of life of patients and their families facing the problems associated with life-threatening illness, through the prevention and relief of suffering by means of early identification and impeccable assessment and treatment of pain and other problems, physical, psychosocial, and spiritual" [3]. What providers, along with patients and family members fail to realize is that PC aims to relieve suffering in all stages of diseases and is not limited to end of life care [2].

\section{Palliative Care Services}

PC teams provide many services to patients and their families. For one, PC teams work closely with patients and family members to establish goals of care during a hospitalization. This includes informing patients and family members on all care plans and appropriate and beneficial medical care [3]. Psychosocial, spiritual, and bereavement support are also key components in PC [3]. When patients are seriously ill, family members face major challenges in sorting out and understanding the medical plan for their loved ones. Nonetheless, PC teams assist families in understanding and regaining control over the patient's care and also receiving further care in the most appropriate setting with the most appropriate needs and resources [3]. PC services utilize a team of experts to achieve the best quality of life for patients including their physical, psychological, social, and spiritual aspects [4].

\section{Qualifications for a Palliative Care Consult}

Emergency providers have a hard time determining which patients qualify for a palliative care consult (PCC) while working in a high pace environment. With that said, there have been multiple criteria that have developed over the years to help providers sort out patients that qualify for a PCC and patients that do not. The National Comprehensive Cancer Network released guidelines on PC which included screening patients with: uncontrolled symptoms, moderate to severe distress related to their diagnosis and treatment, serious comorbidities, and life expectancy less than or equal to six months [3]. Other proposed criteria include: spiritual or existential crisis, delirium, spinal cord compressions, brain injuries, or advanced cancer [3].

\section{Palliative Care in the Emergency Department}

ED physicians often believe the ER is not an ideal environment to deliver PC services, however, the ED presents a key decision point at which providers set the course for a patient's subsequent trajectory and goals of care [4]. The goal of emergency medicine is to provide initial and definitive treatment to all patients. However, what happens if patients present with advanced malignancies or chronic, life threatening illnesses that require complex management? Chronic diseases are now the leading causes of death, and there continues to be a high number of physical, psychosocial, spiritual, and financial suffering associated 
with serious and complex illnesses [4]. Nonetheless, movement in educating emergency providers, hospitalists, and intensivists from acute aggressive interventions to patient-goal assessment continues [2]. This movement in emergency care will reduce the burdensome of patients' symptoms, while also improving the quality of life in patients [5]. More recently there has been an initiation of pilot programs to deliver ED-based PCC. The PC programs have been initiated by the PC team, emergency physicians, or PC champions in the ED. "Some programs have focused on the provision of hospice services through partnerships with hospice providers, which can potentially help ED providers with disposition" [4]. These programs have also allowed a number of emergency physicians to be certified in both disciplines, emergency medicine and PC, in which they have the opportunity to act as champions in the ED [4].

\section{Current Outcomes}

Initiating PC in the ED may lead to positive outcomes such as patient and family satisfaction, reduction in length of stay, and lessening hospital costs. Salins, Deodhar, and Muckaden (2016) analyzed a review of literature that looked at determining factors influencing family satisfaction with PC teams. Findings indicated a positive influence when communication, family support, and end of life care support was provided to patients and their families from healthcare providers [6]. In addition, three different studies examined the effects of proactive PCC on length of stay for high-risk patients in an ICU. The study conducted by Norton et al. (2007) demonstrated an association with significantly shorter ICU length of stays in high risk groups without any significant differences in mortality rates or discharge disposition when PC was initiated early [7]. The second study looked at the outcomes after PCC were initiated early. With early initiation of PCC, a trend displayed PC consultations reduced length of stay and costs without impacting a patient's mortality [8]. Lastly, Khandelwal et al. (2016) discovered $26 \%$ relative risk reduction in length of stay with PC interventions [9]. Although these studies took place in the ICU, the findings are important for emergency medicine because it presents a trend that initiation of PC leads to better patient outcomes. A study in New York examined medical data to determine the effect on hospital costs of PC team consultations for patients enrolled in Medicaid at four different hospitals [10]. Results displayed a consistent decrease of around $\$ 6900$ in hospital costs during a given admission when patients received a PCC early on in their hospitalization [10]. Moreover, inpatient PCC programs have also shown to save hospital money and provide clear patterns of cost saving money [11]. Future research is needed to expand the scope of economic evaluation on this field, specifically focusing in on emergency medicine and to further understand the role of PC enhancing value in health care [11].

\section{Gap of Knowledge}

Despite clear established benefits of PC in the ED, PC continues to be imple- 
mented late in a patient's illness [1]. One of the gaps stems from the lack of PCC in the outpatient settings. "Large gaps in the delivery of PC services exist in the outpatient setting, where there is a failure to address goals of care and to plan for and treat predictable crises" [4]. Another gap is the misconception of PCsolely for patients near death. A study by Smith et al. (2009) examined provider perspectives on improving $\mathrm{PC}$ in the ED. The findings revealed that providers equate PC with end of life care [12]. Providers also disagreed about the feasibility and desirability of providing PC in the ED. Participants agreed that there was a lack of communication between outpatient and ED providers, leading to undesirable outcomes [12]. Another barrier noted from emergency physicians is the difficulty introducing PC to patients and family members that come from different cultures, speak different languages, and have various religious beliefs [1]. Fassier et al. (2016) qualitatively explored the physicians' perceptions of and attitudes toward triage and end of life decisions for elderly critically ill patients in the ED. Results included common themes like negative and positive stereotypes and biases based on one's physiologic age, which influenced the physicians' decision making in resuscitate/do not resuscitate situations [13]. The last study on physicians' perceptions on PC services found common themes. Although providers acknowledged the benefits of PC presence in the ED, they believed the concerns about medicolegal issues impaired their ability to forgo treatments where risks outweigh benefits [14]. The participants also felt that it was ultimately the primary physician's responsibility to address goals of care, as the ED culture stabilizes patients that present with acute medical emergencies [14]. Lastly, critical care literature reported ineffective communication between ED and ICU physicians as one of the most significant barriers for initiating PCC [15]. According to Fassier et al. (2016) emergency physicians tend to trigger ICU admissions and impose disproportionate intensive care on dying patients, beyond individual end of life preferences. This leaves ICU physicians with the need to also perform aggressive care on patients, without even addressing the patient's wants and needs.

\section{Recommendations}

Initiation of PC plays a big role in emergency medicine as patients are being admitted to the ER with more complex diseases, requiring much more than acute stabilization [2]. Future research and collaboration within the ER needs to be a priority to set standards, education, and further models to help emergency physicians. Recent focus on PC has led medical schools worldwide to grow their PC curricula [16]. They suggested integrating developmentally appropriate, basic PC competencies into each year of the medical school curriculum to avoid both neglect of the topic and educational overload [16]. The Liaison Committee for Medical Education in the United States also required all medical schools to include education in PC and end-of-life care. The Medical School Objectives Project identified "knowledge of the major ethical dilemmas in medicine, particularly those that arise at the beginning and end of life" and "knowledge about 
relieving pain and ameliorating the suffering of patients" as outcomes that all medical students should have achieved by graduation" [17]. Goldonowicz, Runyon, and Bullard (2018) designed and implemented a simulation program for their third year ED residency program, in which residents participated in simulation sessions that highlighted complex patient cases with PC focus. A 45 min debriefing session took place after the simulation, and all participants completed an anonymous pre and post education intervention survey [18]. After the intervention, there was an overall confidence in implementing PC skills and perceived importance of PC in the ER setting [18]. Residents felt that emergency medicine providers play an important role in PC and felt that simulation was an effective means to learn PC [18]. While establishing set curriculums in medical school on PC has received some recognition, future studies are still needed to improve the long-term outcomes on collaborating with institutions, assessing higher level of outcomes, skills, and behaviors towards PC in the ED setting [16].

\section{Discussion and Nursing Role}

"The emergency department offers a promising setting to connect admitted patients with PC early on in their hospitalizations" [19]. While ER physicians are recognizing the importance and positive outcomes that arise from early initiation of PC, the questions of when and who will initiate it still remains. Kistler et al. (2015) revealed that $51 \%$ of admitted patients receiving PCC at a medical center were admitted through the ER, but only $6 \%$ of consultations were initiated by an emergency physician [19]. The rising role of a NP could be that key figure for early initiation in the ER. Advanced practicing nurses play a critical role in healthcare especially in the hospital setting. According to the American Association of Nurse Practitioners (2018), "NPs are quickly becoming the health partner of choice for millions of Americans. As clinicians that blend clinical expertise in diagnosing and treating health conditions with an added emphasis on disease prevention and health management, NPs bring a comprehensive perspective and personal touch to health care" [20]. A study conducted by Laurant et al. (2008) looked at patients' preference on healthcare providers. The majority of patients were satisfied with both NPs and physicians; however, patients were more satisfied by the NP in regard to certain aspects of care including support, comfort, and patient goals for both them and their relatives [21]. The study also found that NPs spent an average of 24 minutes per consultation which was two to three times longer than general practitioners [21]. NP programs are also starting to include PC into their curriculum and believe it is essential for their nursing practice [22]. NPs have a foundation of providing communication and care towards their patients and their families because of their holistic approach. Having that foundation and experience sets them up for impacting the world of PC. With the start of adding PC curriculum into advanced practice nurse programs, and the role NPs now have in healthcare, this may be the start of solving the lack of PC initiation that occurs in emergency medicine. With the presence 
of the NP continuing to rise in healthcare, we may start to see a rise in early initiation of PC during a patient's hospitalization course.

\section{Conclusion}

Current knowledge reveals the positive outcomes of early initiation of palliative care such as ensuring patient's quality of life is met, improving education and communication among family members, decreasing length of stay, and improving hospital costs. However, there continues to be a lack of early PC in the ED which may lead to poor patient outcomes. Previous findings indicated that providers continue to have a difficult time implementing palliative care into emergency medicine. Though new medical programs are providing training in PC, the case for an advanced practice nurse is strong as patients may even prefer NPs as they spend more time with patients and receive PC training during their schooling. Future research should examine the role of an NP in providing PC in the ED as evidence supports the need to benefit both the hospital and patients. Future research and collaboration with NPs within the ER also need to be a priority to set standards, education, and further models to help emergency physicians implement palliative care into their practice.

\section{Conflicts of Interest}

The authors declare no conflicts of interest regarding the publication of this paper.

\section{References}

[1] Perrin, K.O. and Kazanowski, M. (2015) Overcoming Barriers to Palliative Care Consultation. Critical Care Nurse, 35, 44-52. https://doi.org/10.4037/ccn2015357

[2] Mierendorf, S.M. and Gidvani, V. (2014) Palliative Care in the Emergency Department. The Permanente Journal, 18, 77. https://doi.org/10.7812/TPP/13-103

[3] Meier, D.E. and McCormick, E. (2015) Benefits, Services, and Models of Subspecialty Palliative Care. UpToDate.

[4] Grudzen, C.R., Stone, S.C. and Morrison, R.S. (2011) The Palliative Care Model for Emergency Department Patients with Advanced Illness. Journal of Palliative Medicine, 14, 945-950. https://doi.org/10.1089/jpm.2011.0011

[5] Atreya, S. (2016) Early Integration of Palliative Medicine into Emergency Care: Is it a Feasible Option. Indian Journal of Medical and Paediatric Oncology. Official Journal of Indian Society of Medical \& Paediatric Oncology, 37, 202. https://doi.org/10.4103/0971-5851.190360

[6] Salins, N., Deodhar, J. and Muckaden, M.A. (2016) Intensive Care Unit Death and Factors Influencing Family Satisfaction of Intensive Care Unit Care. Indian Journal of Critical Care Medicine: Peer-Reviewed, Official Publication of Indian Society of Critical Care Medicine, 20, 97. https://doi.org/10.4103/0972-5229.175942

[7] Norton, S.A., et al. (2007) Proactive Palliative Care in the Medical Intensive Care Unit: Effects on Length of Stay for Selected High-Risk Patients. Critical Care Medicine, 35, 1530-1535. https://doi.org/10.1097/01.CCM.0000266533.06543.0C

[8] Kyeremanteng, K., et al. (2018) The Impact of Palliative Care Consultation in the 
ICU on Length of Stay: A Systematic Review and Cost Evaluation. Journal of Intensive Care Medicine, 33, 346-353. https://doi.org/10.1177/0885066616664329

[9] Khandelwal, N., et al. (2015) Estimating the Effect of Palliative Care Interventions and Advance Care Planning on ICU Utilization: A Systematic Review. Critical Care Medicine, 43, 1102. https://doi.org/10.1097/CCM.0000000000000852

[10] Morrison, R.S., et al. (2011) Palliative Care Consultation Teams Cut Hospital Costs for Medicaid Beneficiaries. Health Affairs, 30, 454-463. https://doi.org/10.1377/hlthaff.2010.0929

[11] May, P., Normand, C. and Morrison, R.S. (2014) Economic Impact of Hospital Inpatient Palliative Care Consultation: Review of Current Evidence and Directions for Future Research. Journal of Palliative Medicine, 17, 1054-1063. https://doi.org/10.1089/jpm.2013.0594

[12] Smith, A.K., et al. (2009) Am I Doing the Right Thing? Provider Perspectives on Improving Palliative Care in the Emergency Department. Annals of Emergency Medicine, 54, 86-93.

[13] Fassier, T., et al. (2016) Who Am I to Decide Whether This Person Is to Die Today? Physicians' Life-or-Death Decisions for Elderly Critically Ill Patients at the Emergency Department-ICU Interface: A Qualitative Study. Annals of Emergency Medicine, 68, 28-39. e3.

[14] Grudzen, C.R., et al. (2012) Does Palliative Care Have a Future in the Emergency Department? Discussions with Attending Emergency Physicians. Journal of Pain and Symptom Management, 43, 1-9.

https://doi.org/10.1016/j.jpainsymman.2011.03.022

[15] Limehouse, W.E., et al. (2012) A Model for Emergency Department End-of-Life Communications after Acute Devastating Events-Part II: Moving from Resuscitative to End-of-Life or Palliative Treatment. Academic Emergency Medicine, 19, 1300-1308. https://doi.org/10.1111/acem.12018

[16] DeCoste-Lopez, J., Madhok, J. and Harman, S. (2015) Curricular Innovations for Medical Students in Palliative and End-of-Life Care: A Systematic Review and Assessment of Study Quality. Journal of Palliative Medicine, 18, 338-349. https://doi.org/10.1089/jpm.2014.0270

[17] Von Gunten, C.F., et al. (2012) Development and Evaluation of a Palliative Medicine Curriculum for Third-Year Medical Students. Journal of Palliative Medicine, 15, 1198-1217. https://doi.org/10.1089/jpm.2010.0502

[18] Goldonowicz, J.M., Runyon, M.S. and Bullard, M.J. (2018) Palliative Care in the Emergency Department: An Educational Investigation and Intervention. BMC Palliative Care, 17, 43. https://doi.org/10.1186/s12904-018-0293-5

[19] Kistler, E.A., et al. (2015) Emergency Department-Triggered Palliative Care in Advanced Cancer: Proof of Concept. Academic Emergency Medicine, 22, 237-239. https://doi.org/10.1111/acem.12573

[20] Practintioners A.A.o.N. (2018) What's a Nurse Practitioner (NP)? https://www.aanp.org/about/all-about-nps/whats-a-nurse-practitioner

[21] Laurant, M.G., et al. (2008) An Overview of Patients' Preference for, and Satisfaction with, Care Provided by General Practitioners and Nurse Practitioners. Journal of Clinical Nursing, 17, 2690-2698. https://doi.org/10.1111/j.1365-2702.2008.02288.x

[22] Rosenzweig, M., et al. (2008) Patient Communication Simulation Laboratory for Students in an Acute Care Nurse Practitioner Program. American Journal of Critical Care, 17, 364-372. 